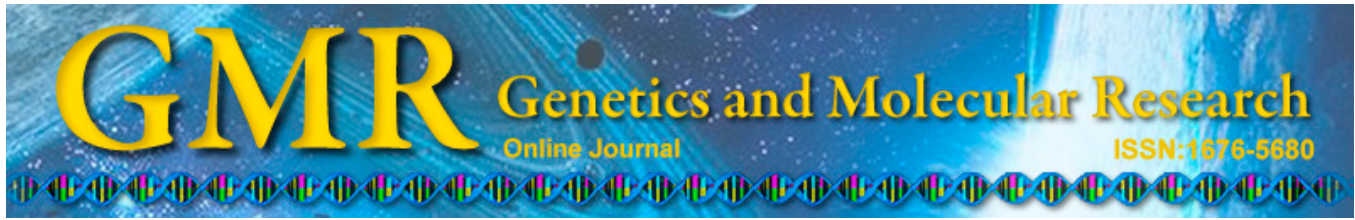

\title{
Rifampicin resistance among multi-resistant MRSA clinical isolates from Chennai, India, and their molecular characterization
}

\author{
K. Murugan ${ }^{1,2}$, K. Kavitha ${ }^{2}$ and S. Al-Sohaibani ${ }^{1}$ \\ ${ }^{1}$ Department of Microbiology and Botany, College of Science, \\ King Saud University, Riyadh, Saudi Arabia \\ ${ }^{2}$ Research and Development Center, Bharathiar University, \\ Coimbatore, Tamil Nadu, India \\ Corresponding author: K. Murugan \\ E-mail:murutan@gmail.com
}

Genet. Mol. Res. 14 (1): 2716-2725 (2015)

Received March 26, 2014

Accepted November 11, 2014

Published March 31, 2015

DOI http://dx.doi.org/10.4238/2015.March.31.1

\begin{abstract}
High-level methicillin-resistant Staphylococcus aureus (MRSA) isolates show rapid evolution of rifampicin resistance, forcing reliance upon expensive and often inferior antibiotics to manage these infections. Accordingly, this study was conducted to: 1) evaluate the level of multidrug resistance among hospital-associated MRSA isolates from Chennai, India; 2) determine their rifampicin resistance and molecular characterization; and 3) analyze the identified rроB gene mutations for predominant high-level rifampicin resistanceassociated nucleotide changes. Conventional laboratory techniques and antibiogram evaluation by disc diffusion were utilized for isolate phenotypic identification. Among the 54 isolates obtained, 74\% (42) were found to be MRSA. All the isolates exhibited complete susceptibility to linezolid and vancomycin, and variable resistance to conventional antibiotics; the MAR index value calculated was 0.64 . Genotypic identification of the high-level rifampicin-resistant isolate $S$. aureus KM05 was established through rpoB amplification and
\end{abstract}


sequencing. The evolutionary relationship of KM05 to other isolates and its $r p o B$ gene mutation status was determined to understand the genetic basis of its high rifampicin resistance. The $S$. aureus KM05 rрo $B$ gene yielded $\geq 98 \%$ sequence similarity and a close phylogenetic relationship with other known reference database organisms. It also showed mutational changes in three rpoB codon positions encoding amino acids at positions 455, 481, and 529. These results have established the prevalence of rifampicin resistance among Chennai hospital MRSA isolates, and suggest that the predominant high-level resistance nucleotide changes would serve to form a basis for their diagnosis and control of rifampicin-resistant MRSA.

Key words: Genotype; MRSA; Rifampicin; rpoB; Staphylococcus aureus

\section{INTRODUCTION}

Emergence of antibiotic resistance to existing therapeutic agents and the circulation of genetic variants that escape immune surveillance limit the efficacy of currently employed infectious disease therapeutic and prophylactic interventions. In addition, previous inadequate scientific approaches warrant new research strategies and tactics to minimize these threats and challenges for improving global health (Murugan et al., 2013). Currently, the high prevalence of common pathogen antibiotic resistance and their increasing threat to public health is a growing concern among medical practitioners. This resistance can occur through number of varied mechanisms, including target modification, acquisition of resistance-conferring genes, spontaneous mutations, and upregulation of genes encoding cellular efflux pumps. Knowledge of these mechanisms can provide a better understanding of novel ways to treat antibiotic resistance (Beach and Champney, 2014).

Staphylococcus aureus is the leading etiological agent of many mild to severe bloodstream, lower respiratory tract, and skin and soft tissue bacterial infections in many developed countries (Lindsay, 2014). Methicillin-resistant S. aureus (MRSA) strains have grown into one of the major health threats in the United States as well as globally during the last two decades. MRSA strains alone have caused over half of all staphylococcal infections, whose death toll exceeded even that from HIV/AIDS in the United States during 2005 (Patel et al., 2013). The United Kingdom recorded the highest MRSA rates among European countries (Edmiston et al., 2013) and in the United States MRSA is probably the leading cause of death by any single infectious agent (Lindsay, 2014). MRSAs are known for life-threatening pneumonias and infections of the bloodstream and surgical sites in healthcare facilities, and the inability of $\beta$-lactam antibiotics to prevent or treat these infections has forced the medical community to rely on expensive and often inferior antibiotics (Lindsay, 2014). The consequences of MRSA-implicated surgical site infections were catastrophic both for patients and for the use of healthcare resources. These MRSA-caused infections led to a longer postoperative stay, high treatment costs, and a poorer prognosis. Their increasing rate and prevalence have stimulated an accelerated interest in the preoperative identification of MRSA colonization in surgical patients (Edmiston et al., 2013).

The chemical structure of rifampicin allows it to penetrate well into tissues and abscesses, which are poorly penetrated by most other antistaphylococcal agents (Darley and MacGowan, 2004). These broad-spectrum antibiotics exert their activity by interacting spe- 
cifically with the $r p o B$ gene encoding the $\beta$-subunit of the bacterial RNA polymerase (Dey et al., 2011). Mutations in rроB genes of Staphylococcus spp and other bacterial species mainly consist of single nucleotide polymorphisms, which lead to the rapid emergence of resistance (Hellmark et al., 2009).

Rifampicin resistance in $S$. aureus, as in other bacteria, is associated with mutations in particular regions (cluster I and II) of rpoB (Zhou et al., 2012). Aiba et al. (2013) proposed that a regulatory mutation of $r р о B$ influences the susceptibility of the cell to vancomycin, daptomycin, and linezolid. Mutations also influence $\beta$-lactam resistance, as well as conferring homogeneous methicillin resistance so that all the mutant strains in a cell population uniformly express high resistance to $\beta$-lactam antibiotics and are comprised of the peculiar Eagletype, leading to better growth of $S$. aureus cells on high-concentration methicillin agar plates than on low-concentration plates, depending on the mode of expression of the mecA gene, which encodes the $\beta$-lactam lower affinity penicillin-binding protein (PBP2 $\alpha$ ). Eagle type is a resistant phenotype manifesting the expression of chromosomal mutation chr+ required for the hetero-to-homo phenotypic conversion equivalent genetic alteration under mecI-mediated repression of PBP $2 \alpha$ production (Kondo et al., 2001). Since rifampicin in combination therapy with other antibiotics evidently results in rapid emergence of resistance, continuous and thorough monitoring for the emergence of resistance remains essential. $r p o B$ gene sequencing is a rapid, objective, and accurate method of simultaneous species identification and detection of rifampicin resistance in staphylococci (Hellmark et al., 2009). It is of high importance to characterize MRSA resistant phenotypes and genotypes with reference to their rpoB gene mutation for effective control of resurgent epidemics. Although reports are available on the rpo $B$ gene mutations in the tuberculosis-causative Mycobacterium tuberculosis northern (Sharma et al., 2003) and southern (Mani et al., 2001) Indian isolates, the literature is silent on the equally threatening MRSA isolates. Hence, the objectives of the present study included: 1) phenotypic and genotypic characterization of multi-resistant surgical-site MRSA isolates to determine whether they reflect independently arisen novel genotypes or the spread of current representatives of previously detected genotypes; and 2) to analyze high-level rifampicin resistanceassociated predominant nucleotide changes in the $r p o B$ gene.

\section{MATERIAL AND METHODS}

\section{Sample collection and isolation}

For the evaluation of phenotypic rifampicin and other antibiotic resistant phenotypes and $r р о B$ gene sequencing, clinical $S$. aureus species were isolated from different hospital intensive care unit (ICU) environment samples in Chennai, India. Aerobic cultures were obtained by swabbing the abscess cavity with a cotton-tipped culture swab moistened in sterile saline solution. Specimens were placed in a sterile container with $2 \mathrm{~mL}$ sterile Stuart's medium (HiMedia, Mumbai, India), transported to the laboratory, and processed in accordance with National Committee for Clinical and Laboratory Standards (NCCLS, 2003) methodology. Swab samples were streaked directly on mannitol salt agar medium containing (per liter): proteose peptone (10 g), beef extract (1 g), D-mannitol (10 g), sodium chloride (75 g), phenol red $(25 \mathrm{mg})$, and agar $(15 \mathrm{~g}), \mathrm{pH} 7.4 \pm 0.2$ (HiMedia), and incubated at $37^{\circ} \mathrm{C}$ for $24-48 \mathrm{~h}$. Yellow-colored colonies presumptively identified as coagulase-positive $S$. aureus were isolated and subjected to routine diagnostic procedures. Briefly, Gram staining, catalase, oxidase, 
indole, nitrate reduction, methyl red, Voges-Proskauer, sugar fermentation, DNAse, as well as coagulase tests were performed to substantiate isolate identification.

\section{Determination of antibiogram}

The antimicrobial susceptibility and resistance of all isolated $S$. aureus isolates were determined using the disc diffusion assay on Mueller-Hinton agar containing (per liter) beef infusion (300 g), casein acid hydrolysate (17.5 g), starch (1.5 g), and agar (15 g), pH $7.4 \pm 0.2$ (HiMedia) according to the methodology of NCCLS (2003). The antimicrobial agents were chosen on the basis of their importance in treating human or animal infections and were purchased from HiMedia. Antimicrobial discs having the following concentrations were used: ampicillin $(10 \mu \mathrm{g})$, amoxicillin clavulanic acid $(30 \mu \mathrm{g})$, chloramphenicol $(30 \mu \mathrm{g})$, ciprofloxacin $(10$ $\mu \mathrm{g})$, clindamycin $(2 \mu \mathrm{g})$, co-trimoxazole $(25 \mu \mathrm{g})$, erythromycin $(15 \mu \mathrm{g})$, fusidic acid $(10 \mu \mathrm{g})$, gentamicin $(10 \mu \mathrm{g})$, linezolid $(10 \mu \mathrm{g})$, nalidixic acid $(30 \mu \mathrm{g})$, norfloxacin $(10 \mu \mathrm{g})$, oxacillin $(1$ $\mu \mathrm{g})$, penicillin $(10 \mathrm{U})$, tetracycline $(30 \mu \mathrm{g})$, and vancomycin $(30 \mu \mathrm{g})$. The multiple antibiotic resistance (MAR) index (Olayinka et al., 2004) was calculated using the formula described by Hung et al. (2012), below. The rifampicin minimum inhibitory concentrations (MICs) of all isolates were determined by the agar dilution method. The isolates were interpreted as rifampicinsensitive (MIC $\leq 1 \mathrm{mg} / \mathrm{L}$ ) or -resistant (MIC $\geq 4 \mathrm{mg} / \mathrm{L}$ ) as per CLSI criteria (Zhou et al., 2012).

$$
\text { MAR Index }=\mathrm{y} / \mathrm{nx}
$$

where $y=$ total number of resistant colonies scored; $n=$ number of isolates; and $x=$ total number of antibiotics tested.

\section{Genotypic detection of rifampicin resistance-associated mutations}

\section{DNA preparation}

The extraction and purification of genomic DNA from the rifampicin-resistant MRSA isolate $S$. aureus KM05 were performed by following a modified cetyl trimethyl ammonium bromide-sodium chloride method. The purified DNA pellet was washed with $70 \%$ ethanol three times, dried, and stored at $-20^{\circ} \mathrm{C}$ after resuspension, as described previously (Natarajaseenivasan et al., 2012). The concentration of the extracted DNA and its purity were determined by measuring the 260/280 nm optical density ratios using a GENESYS 10S Bio UV/Vis spectrophotometer (Thermo Fisher Scientific Inc., Waltham, MA, USA). Following electrophoresis on a $0.8 \%$ agarose gel, the ethidium bromide-stained DNA band was used to determine the integrity of the genomic DNA.

\section{rpoB gene amplification and sequencing}

The oligonucleotide primers 5'-TCT TTA TGG GTG ATT TCC CA-3' and 5'-ACC GTC GTT TAC GTT CTG TA-3' (Didier et al., 2011) were utilized for the amplification of rifampin resistance clusters I and II encompassing the $r p o B$ gene sequence. Polymerase chain reaction (PCR) was carried out in a $50-\mu \mathrm{L}$ volume containing $6.25 \mu \mathrm{L} 0.35 \mu \mathrm{M}$ of each primer, $6.25 \mu \mathrm{L} 0.25 \mathrm{mM}$ of each dNTP (dATP, dCTP, dGTP, and dTTP), $2.5 \mu \mathrm{L} 100 \mathrm{ng}$ DNA tem- 
plate, $1.5 \mu \mathrm{L} 1.5 \mathrm{mM} \mathrm{MgCl}, 0.55 \mu \mathrm{L}$ 1U Prime Taq DNA polymerase (Genetbio, Daejeon, Korea), $5 \mu \mathrm{L} 1 \mathrm{X}$ Taq buffer, and $21 \mu \mathrm{L}$ MilliQ water. The PCRs were performed in a DNA thermal cycler (Eppendorf; Hamburg, Germany). The PCR cycling programs consisted of an initial denaturation $\left(4 \mathrm{~min}\right.$ at $\left.94^{\circ} \mathrm{C}\right)$ followed by 40 cycles of denaturation $\left(1 \mathrm{~min}\right.$ at $\left.94^{\circ} \mathrm{C}\right)$, annealing $\left(1 \mathrm{~min}\right.$ at $\left.55^{\circ} \mathrm{C}\right)$, and extension $\left(1 \mathrm{~min}\right.$ at $\left.72^{\circ} \mathrm{C}\right)$, with a final extension $\left(7 \mathrm{~min}\right.$ at $\left.72^{\circ} \mathrm{C}\right)$. The amplicon of the partial cluster I gene was purified using a QIAquick PCR purification kit (Qiagen, Madrid, Spain). The sequences of the purified amplicons were obtained by Sanger dideoxy sequencing, carried out by Ocimum Biosolution (HITEC City, India) using an ABI 3130 genetic analyzer (Applied Biosystems, Foster City, CA, USA). The rpoB sequence was submitted to the National Center for Biotechnology Information (NCBI) and has been assigned the accession No. KJ559410.

\section{Genotypic identification, phylogenetic and mutation analysis}

For genotypic identification, the sequence data of the $S$. aureus KM05 rpoB gene amplicons were compared with reference and closest bacterial species sequence data from GenBank using the nucleotide-nucleotide BLAST program (http://www.ncbi.nlm.nih.gov/) (Hauschild et al., 2010). The phylogenetic relationship of the $S$. aureus KM05 rpoB gene with other $S$. aureus sequences in the reference database was inferred by the neighbor-joining method (Saitou and Nei, 1987) using the Molecular Evolutionary Genetics Analysis software version 4.0 (MEGA4) (Tamura et al., 2007). An optimal phylogenetic tree was drawn and the evolutionary distances were computed using the maximum composite likelihood method (Tamura et al., 2004), which is given in units of the number of base substitutions per site. All positions containing gaps and missing data were eliminated from the final dataset, which comprised a total of 335 positions. The $S$. aureus KM05 rpoB gene nucleotide sequence obtained was compared with the wild type $S$. aureus subsp aureus rpoB gene sequence (GenBank accession No. CP003033.1) using the pairwise sequence alignment of DNA sequences from EMBOSS needle, which incorporates the global pairwise sequence alignment Needleman-Wunsch algorithm (http://emboss.open-bio.org/).

\section{RESULTS}

A total of 54 strains presumptively identified as $S$. aureus were isolated from swab samples from patients admitted to the ICUs of various Chennai hospitals during 2013. Through standard conventional bench top phenotypic culture and biochemical characterization tests (Table 1), the isolates were identified and their MRSA status determined. Among the 54 isolates obtained, 42 were found to be MRSA. The antibiogram study and the calculated isolate susceptibility percentage showed that all MRSA isolates exhibited $100 \%$ resistance to ampicillin, amoxicillin, clavulanic acid, fusidic acid, nalidixic acid, oxacillin, and penicillin, followed by norfloxacin $(96 \%)$, ciprofloxacin $(61 \%)$, erythromycin $(48 \%)$, gentamicin $(43 \%)$, co-trimoxazole (42\%), tetracycline (31\%), chloramphenicol (4.8\%), and clindamycin $(4.8 \%)$. All the isolates were susceptible to linezolid and vancomycin. The obtained isolate MAR index value was calculated as 0.64 . Of the 44 MRSA strains, 32 were identified as rifampicin-sensitive and 12 as rifampicin-resistant $(28.57 \%)$. Among the latter, the isolate $S$. aureus KM05 showed high-level resistance (MIC $\geq 8 \mathrm{mg} / \mathrm{L}$ ). 
Table 1. Morphological and biochemical test results of hospital-associated MRSA isolates from Chennai.

\begin{tabular}{lll}
\hline Serial number & Test & Result \\
\hline 1 & Gram staining & Gram-positive \\
2 & Motility & Non-motile \\
3 & Indole & Negative \\
4 & Methyl red reaction & Negative \\
5 & Voges-Proskauer reaction & Negative \\
6 & Citrate test & Positive \\
7 & Hydrogen sulfide production & Negative \\
8 & Urease activity & Positive \\
9 & Lactose, glucose, sucrose, & Positive \\
10 & D-Mannitol, trehalose, mannose, galactose & Positive \\
11 & L-Arabinose, raffinose, D-cellubiose & Negative \\
12 & Lipid hydrolysis & Positive \\
13 & Starch hydrolysis & Negative \\
14 & Gelatin liquefaction & Negative \\
15 & Catalase & Positive \\
16 & Oxidase & Negative \\
17 & Growth in NaCl (10\%) & Positive \\
18 & DNAse & Positive \\
19 & Urease & Positive \\
20 & Coagulase & Positive \\
21 & Oxidase & Positive \\
\hline
\end{tabular}

BLAST analysis of rifampicin-resistant MRSA S. aureus KM05 isolate partial rpoB gene sequence (NCBI ID: KJ559410) confirmed its genotypic identity, since it yielded $\geq 98 \%$ sequence similarity with the closest and reference bacterial species GenBank sequences. The evolutionary history of $S$. aureus KM05 inferred using the neighbor-joining method resulted in an optimal tree with the sum of branch lengths $=0.01649896$ (Figure 1). The evolutionary distances were computed using the maximum composite likelihood method and are presented in units of the number of base substitutions per site. The obtained phylogenetic tree analysis of the $S$. aureus KM05 rроB gene showed its close relationship with other isolates and the possibility of horizontal transfer of rpo genes among them.

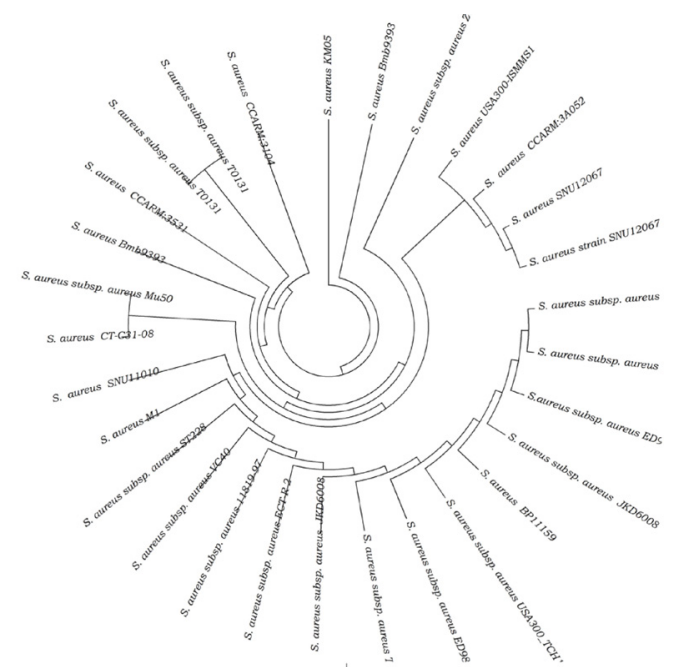

Figure 1. Phylogenetic tree of the rifampicin-resistant MRSA Staphylococcus aureus KM05 isolate, based upon sequence relationships among the rpo genes. 
Mutations in the high-level rifampicin-resistant $S$. aureus KM05 isolates rifampicinresistance determining region of the rроB gene were determined by pairwise sequence alignment of its sequences with that of the wild organism. The rifampicin-resistant MRSA isolate $S$. aureus KM05 harbored point mutations in its $r p o B$ gene at three codon positions, detailed in Table 2. The obtained in silico analysis result (Figure 2) revealed two mutations in cluster I (amino acid positions 450-488) and one mutation in cluster II (amino acid positions 515530 ) of the $S$. aureus KM05 rpoB gene. These mutations are predicted to lead to changes in amino acids at positions 455,481 , and 529. These results suggest that high-level rifampicin resistance of $S$. aureus KM05 isolates is primarily, but not necessarily, associated with these rрoB gene mutations.

Table 2. Mutations found in the rpoB gene of the MRSA isolate Staphylococcus aureus KM05.

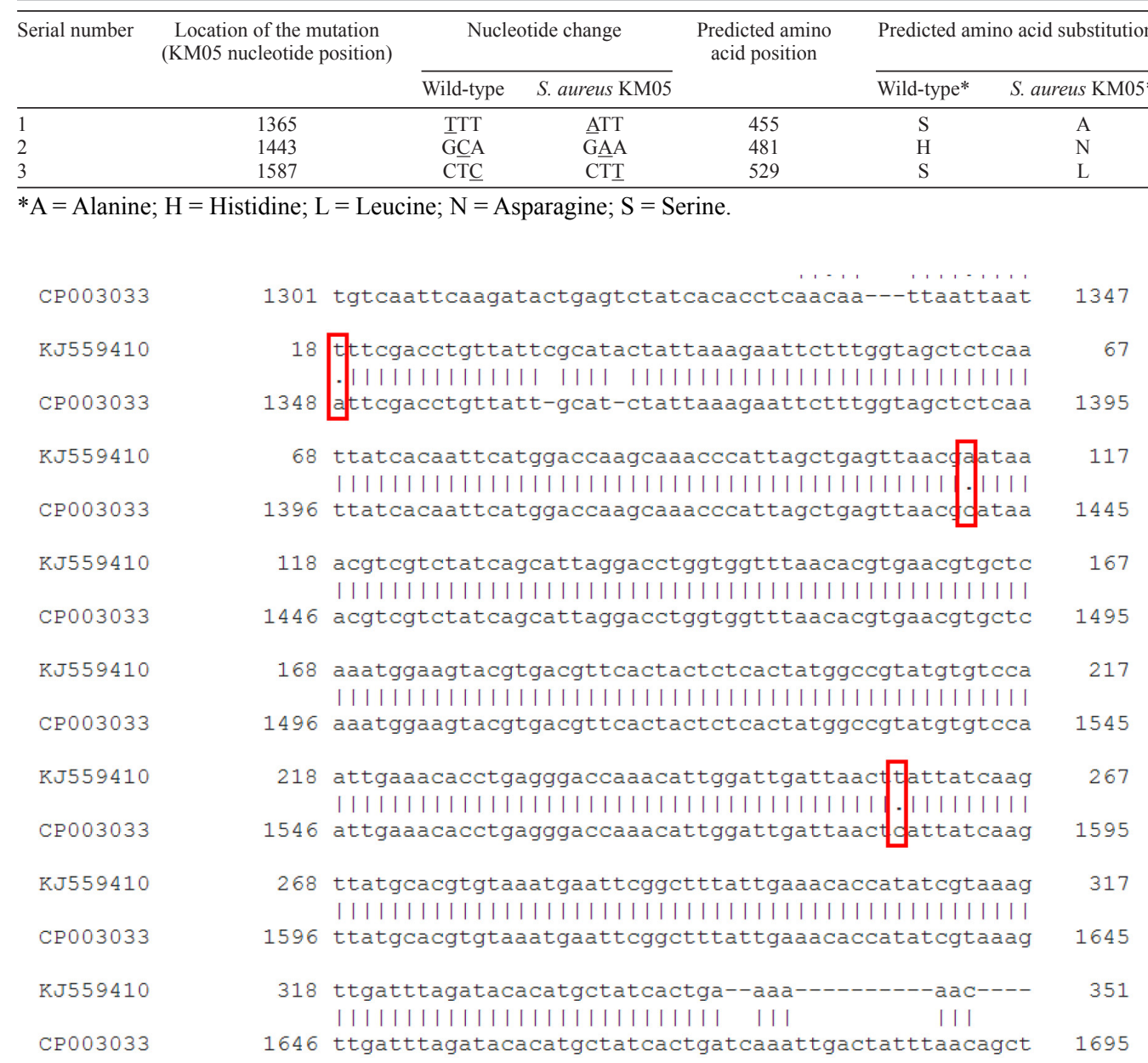

Figure 2. Global pairwise gene alignment showing mutations in the $r p o B$ gene of the rifampicin-resistant MRSA Staphylococcus aureus KM05 isolate. Alignment was performed using EMBOSS needle (http://emboss.open-bio. org/). KJ559410, S. aureus KM05 sequence; CP003033, wild-type $S$. aureus. 


\section{DISCUSSION}

From witnessing the rapid decrease in recent years of the efficacy of antibiotic treatment of common diseases due to the emergence of multi-antibiotic resistance, it has become apparent that we are at the dawn of a post-antibiotic era. Such resistance arises as a result of mutations in microbes as well as selective pressure from antibiotic use, which provides a competitive advantage to resistant organisms (Laxminarayan et al., 2013). An Indian Network for Surveillance of Antimicrobial Resistance (INSAR) study conducted between 2008 and 2009 revealed an endemic high-level MRSA problem in India. This study emphasized the need for studying the epidemiology of such infections, requiring robust antimicrobial stewardship, and strengthening infection control measures for preventing the spread and reducing the emergence of resistance (INSAR, 2013). Hospital-associated MRSAs (HA-MRSAs) are accountable for the high proportion of $S$. aureus infections in many parts of the world. HA-MRSA is expected to continue evolving new virulence and resistance pathways, often by exchanging mobile genetic elements, and in response to local selective pressures. Only a handful of successful HA-MRSA clones are responsible for the majority of infections, whereas different clones dominate across diverse geographical locations (Lindsay, 2014). Resistant organisms implicated in eventual untreatable infections warrant early characterization to arrest and/or delay their spread (INSAR, 2013). The INSAR (2013) study showed higher MRSA isolation rates from ICUs and wards than were seen among outpatients from various hospitals across the country. The present finding of a 77\% MRSA distribution among hospital ICU isolates corroborates with the number of reports of Indian HA-MRSA distributions (Anupurba et al., 2003; Thati et al., 2011). Therefore, the results obtained here on HA-MRSA are no exception to those already reported. The antibiograms of the MRSA isolates obtained showed their complete resistance to $\beta$-lactam ampicillin, amoxicillin, clavulanic acid, penicillin, and oxacillin along with the quinolone nalidixic acid. The conventional antibiotic multidrug resistance shown by the MRSA isolates warrants the need for reviewing the antibiotics selected for their empirical treatment. These isolates showed high resistance to erythromycin (48\%), but low resistance to clindamycin (4.3\%). This macrolide and the lincosamide (clindamycin) resistance can be explained by their altered $23 \mathrm{~S}$ ribosomal RNA, which would result in macrolide lincosamide streptogramin B resistance, which could be either constitutively or inducibly expressed. Erythromycin is known as a more potent inducer of resistance (Natarajaseenivasan et al., 2012). Furthermore, the calculated MAR index value of 0.64 indicates that the isolate originates from an environment where the usage of the antibiotic is frequent (Paul et al., 2002; Bongiorno et al., 2010). Since the MAR value can be used to indicate the extent of microbial exposure to antibiotics used within the community, the value obtained highlights the importance of judicial use of antibiotics for MRSA treatment.

Determination of the exact identification of bacterial pathogens through characterization of their phenotypic properties and 16S-23S rDNA intergenic spacer region polymorphism comprise an essential prerequisite for MRSA management from an epidemiological point of view, especially in situations when monitoring the spread of pathogens is difficult. The application of PCR amplicon sequencing-based methods and/or restriction fragment length polymorphism (RFLP) analysis of PCR products would be a preferred approach (Hauschild et al., 2010). Previously, Marty et al. (2012) reported that these highly discriminatory rрoB species-specific PCR-RFLP analyses allow fast, simple, and accurate molecular identification of Staphylococcus and other bacteria. As the RNA polymerase $\beta$-subunit encoding the rpoB gene has emerged as one of the few potential core gene candidates for phylogenetic analyses 
and identification of bacteria, especially when studying closely related isolates (Adekambi et al., 2009), the present study also supports this paradigm. The genotypic identification of the isolate $S$. aureus KM05 through its $r p o B$ gene confirmed its expressed taxonomic phenotypic characteristics. Hence, the rроB gene can be used as a diagnostic tool for early isolate identification, patient screening, and minimization of MRSA spread.

Mutations in the conserved region of the rpoB gene are known for conferring rifampicin resistance in a number of bacteria, including $S$. aureus. The majority of $S$. aureus rifampicin resistance mutations have been mapped to the conserved rifampicin resistance-determining region of the $r p o B$ gene (Jansen van Rensburg et al., 2012). S. aureus rifampicin resistance is typically due to amino acid substitutions in the three known clusters (I, II, and III) of the RNA polymerase $\beta$-subunit rifampicin-binding site, and the point mutations in clusters I and II are identified as their genetic determinants. Genetic studies carried out to identify catalytically important amino acid changes affecting transcription termination in yeast RNA polymerase III showed that the mutations in regions of the RET1 gene encoding amino acids 455-486 are the termination-altering ones (Shaaban et al., 1995). Furthermore, the high-level rifampicin resistance of the isolate $S$. aureus KM05 might be explained by the previously reported rifampicin high-level resistance-associated amino acid substitution at site 481, one of the most common rifampicin resistance genotypes found herein, which had been reported previously in several laboratory mutants and clinical isolates (Jansen van Rensburg et al., 2012). This substitution leads to disruption of an $\mathrm{H}$ bond between rifampicin and RNA polymerase, and also reduces hydrophobic interactions within the binding cavity, thereby decreasing the affinity of the drug for its target (Jansen van Rensburg et al., 2012). To our knowledge, the amino acid substitutions in amino acid positions 455 and 529 have not been previously reported. In conclusion, this report has established the rifampicin resistance prevalence among the MRSA Chennai hospital isolates, the location and the nature of the amino acid substitutions, and their genetic basis. These findings will likely contribute to the efficient management and control of MRSA.

\section{ACKNOWLEDGMENTS}

Research supported by the King Saud University, Deanship of Scientific Research, College of Science Research Center.

\section{REFERENCES}

Adekambi T, Drancourt M and Raoult D (2009). The rpoB gene as a tool for clinical microbiologists. Trends Microbiol. 17: 37-45.

Aiba Y, Katayama Y, Hishinuma T, Murakami-Kuroda H, et al. (2013). Mutation of RNA polymerase beta-subunit gene promotes heterogeneous-to-homogeneous conversion of beta-lactam resistance in methicillin-resistant Staphylococcus aureus. Antimicrob. Agents Chemother. 57: 4861-4871.

Anupurba S, Sen MR, Nath G, Sharma BM, et al. (2003). Prevalence of methicillin resistant Staphylococcus aureus in a tertiary referral hospital in eastern Uttar Pradesh. Indian J. Med. Microbiol. 21: 49-51.

Beach JM and Champney WS (2014). An examination of the inhibitory effects of three antibiotics in combination on ribosome biosynthesis in Staphylococcus aureus. Arch. Microbiol. 196: 249-260.

Bongiorno D, Campanile F, Mongelli G, Baldi MT, et al. (2010). DNA methylase modifications and other linezolid resistance mutations in coagulase-negative staphylococci in Italy. J. Antimicrob. Chemother. 65: 2336-2340.

Darley ES and MacGowan AP (2004). Antibiotic treatment of Gram-positive bone and joint infections. J. Antimicrob. Chemother. 53: 928-935.

Dey A, Verma AK and Chatterji D (2011). Molecular insights into the mechanism of phenotypic tolerance to rifampicin 
conferred on mycobacterial RNA polymerase by MsRbpA. Microbiology 157: 2056-2071.

Didier JP, Villet R, Huggler E, Lew DP, et al. (2011). Impact of ciprofloxacin exposure on Staphylococcus aureus genomic alterations linked with emergence of rifampin resistance. Antimicrob. Agents Chemother. 55: 1946-1952.

Edmiston CE Jr, Leaper D and Kiernan M (2013). The Yin and Yang of pre-operative screening for meticillin resistant and sensitive Staphylococcus aureus (MRSA and MSSA): Does the extra effort and cost of suppression reduce surgical site infections. Wound Med. 1: 7-12.

Hauschild T, Śliżewski P and Masiewicz P (2010). Species distribution of staphylococci from small wild mammals. Syst. Appl. Microbiol. 33: 457-460.

Hellmark B, Söderquist B and Unemo M (2009). Simultaneous species identification and detection of rifampicin resistance in staphylococci by sequencing of the rpoB gene. Eur. J. Clin. Microbiol. Infect. Dis. 28: 183-190.

Hung WC, Takano T, Higuchi W, Iwao Y, et al. (2012). Comparative genomics of community-acquired ST59 methicillinresistant Staphylococcus aureus in Taiwan: novel mobile resistance structures with IS1216V. PLoS One 7: e46987.

Indian Network for Surveillance of Antimicrobial Resistance (INSAR) group I (2013). Methicillin resistant Staphylococcus aureus (MRSA) in India: prevalence \& susceptibility pattern. Indian J. Med. Res. 137: 363-369.

Jansen van Rensburg MJ, Whitelaw AC and Elisha BG (2012). Genetic basis of rifampicin resistance in methicillin-resistant Staphylococcus aureus suggests clonal expansion in hospitals in Cape Town, South Africa. BMC Microbiol. 12: 46.

Kondo N, Kuwahara-Arai K, Kuroda-Murakami H, Tateda-Suzuki E, et al. (2001). Eagle-type methicillin resistance: new phenotype of high methicillin resistance under mec regulator gene control. Antimicrob. Agents Chemother. 45: 815-824.

Laxminarayan R, Duse A, Wattal C, Zaidi AK, et al. (2013). Antibiotic resistance-the need for global solutions. Lancet Infect. Dis. 13: 1057-1098.

Lindsay JA (2014). Evolution of Staphylococcus aureus and MRSA during outbreaks. Infect. Genet. Evol. 21: 548-553.

Mani C, Selvakumar N, Narayanan S and Narayanan PR (2001). Mutations in the rpoB gene of multidrug-resistant Mycobacterium tuberculosis clinical isolates from India. J. Clin. Microbiol. 39: 2987-2990.

Marty E, Buchs J, Eugster-Meier E, Lacroix C, et al. (2012). Identification of staphylococci and dominant lactic acid bacteria in spontaneously fermented Swiss meat products using PCR-RFLP. Food Microbiol. 29: 157-166.

Murugan K, Sekar K, Sangeetha S and Ranjitha S (2013). In vitro and structure based in silico screening of putative therapeutic quorum sensing inhibitors from an ethanomedicinal herb Achyranthes aspera controlling cariogenic Streptococcus mutans. Pharm. Biol. 51: 728-736.

Natarajaseenivasan K, Shanmughapriya S, Latha R and Artiflavia GB (2012). Prevalence of community acquired methicillin resistant Staphylococcus aureus (CA-MRSA) in skin and soft tissue infections among cases from Puducherry, India. J. Public. Health 20: 593-597.

National Committee for Clinical and Laboratory Standards (NCCLS) (2003). Methods for Dilution Antimicrobial Susceptibility Tests for Bacteria that Grow Aerobically, 6th ed. Approved standard NCCLS document M7-A6. NCCLS, Wayne.

Olayinka AT, Onile BA and Olayinka BO (2004). Prevalence of multi-drug resistance (MDR) Pseudomonas aeruginosa isolates in surgical units of Ahmadu Bello university teaching hospital, Zaria, Nigeria: An indication for effective control measures. Ann. Afr. Med. 3: 13-16.

Patel BA, Ashby CR Jr, Hardej D and Talele TT (2013). The synthesis and SAR study of phenylalanine-derived (Z)-5arylmethylidene rhodanines as anti-methicillin-resistant Staphylococcus aureus (MRSA) compounds. Bioorg. Med. Chem. Lett. 23: 5523-5527.

Paul S, Bezbaruah RL, Roy MK and Ghosh AC (1997). Multiple antibiotic resistance (MAR) index and its reversion in Pseudomonas aeruginosa. Lett. Appl. Microbiol. 24: 169-171.

Saitou N and Nei M (1987). The neighbor-joining method: a new method for reconstructing phylogenetic trees. Mol. Biol. Evol. 4: 406-425.

Shaaban SA, Krupp BM and Hall BD (1995). Termination-altering mutations in the second-largest subunit of yeast RNA polymerase III. Mol. Cell Biol. 15: 1467-1478.

Sharma M, Sethi S, Mishra B, Sengupta C, et al. (2003). Rapid detection of mutations in rpoB gene of rifampicin resistant Mycobacterium tuberculosis strains by line probe assay. Indian J. Med. Res. 117: 76-80.

Tamura K, Nei M and Kumar S (2004). Prospects for inferring very large phylogenies by using the neighbor-joining method. Proc. Natl. Acad. Sci. U. S. A. 101: 11030-11035.

Tamura K, Dudley J, Nei M and Kumar S (2007). MEGA4: Molecular Evolutionary Genetics Analysis (MEGA) software version 4.0. Mol. Biol. Evol. 24: 1596-1599.

Thati V, Shivannavar CT and Gaddad SM (2011). Vancomycin resistance among methicillin resistant Staphylococcus aureus isolates from intensive care units of tertiary care hospitals in Hyderabad. Indian J. Med. Res. 134: 704-708.

Zhou W, Shan W, Ma X, Chang W, et al. (2012). Molecular characterization of rifampicin-resistant Staphylococcus aureus isolates in a Chinese teaching hospital from Anhui, China. BMC Microbiol. 12: 240. 